\title{
Moderate-to-vigorous physical activity, not sedentary time, is associated with total and regional adiposity in a sample of UK adults at risk of type 2 diabetes
}

\author{
D M Harrington ${ }^{1,2,3}, \mathbf{C}$ L Edwardson ${ }^{1,2}$, J Henson ${ }^{2}$, K Khunti ${ }^{1}$, \\ T Yates $^{1,2}$ and M J Davies ${ }^{1,2}$ \\ ${ }^{1}$ Diabetes Research Centre, University of Leicester, Leicester, UK \\ 2 NIHR Leicester-Loughborough Diet, Lifestyle and Physical Activity Biomedical \\ Research Unit, Leicester, UK \\ E-mail: dh204@le.ac.uk
}

Received 2 March 2016, revised 13 June 2016

Accepted for publication 21 July 2016

Published

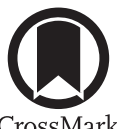

\section{Abstract}

How sedentary time (ST) relates to total and regional adiposity and whether these associations are independent of moderate-to-vigorous physical activity (MVPA) are of clinical and public health interest. We assessed the relationship between objectively measured MVPA, ST and ST breaks in adults at risk of type 2 diabetes (T2D).

The sample consisted of 163 adults (37-75 years) recruited from primary care. Dual energy x-ray absorptiometry (iDXA, GE Corp) was used to estimate total and regional (appendages and trunk) fat mass as well as fat mass in the abdominal (android) and hip/thigh (gynoid) regions. The Actigraph GT3X was worn over $7 \mathrm{~d}$ (participants with $\geqslant 4$ valid days included herein) and published cut-points were applied to $15 \mathrm{~s}$ epoch data to estimate mean MVPA, ST and breaks in ST. Multiple regression investigated associations between both ST and MVPA with total and regional adiposity without and with adjustment for known and potential confounders (including total lean mass) and for each other.

MVPA was negatively and significantly associated with total $(b=-62$; $p=0.002)$, and regional $(p<0.05)$ adiposity before and after adjusting for confounders including ST. Although ST was positively and significantly associated with total $(b=46.4 ; p=0.002)$ and regional adiposity (all $p \leqslant 0.007)$, these relationships disappeared after adjustment for MVPA $(p>0.05)$. Sedentary breaks were not significantly related to adiposity before

\footnotetext{
${ }^{3}$ Author to whom any correspondence should be addressed.

Leicester Diabetes Centre (origin wing), Leicester General Hospital, Leicester LE5 4PW, UK.
} 
or after adjustment. An age-by-MVPA interaction for total, android, gynoid and trunk fat revealed that MVPA did not remain significant when controlling for ST and other covariates in the adults over 65 years old.

In this sample of adults at risk of type 2 diabetes, MVPA, and not ST, was associated with total and regional adiposity after adjustment for each other. However, in older adults at risk of T2D weight related benefits may be gained from reducing daily ST and increasing MVPA.

Keywords: sedentary, physical activity, adiposity

(Some figures may appear in colour only in the online journal)

\section{Introduction}

Sedentary time (ST) has been related to cardiometabolic risk factors, independent of moderateto vigorous-intensity physical activity (MVPA) (Henson et al 2013, Cooper et al 2014, van der Berg et al 2016) and recent systematic reviews have associated ST to diabetes incidence and both specific and all-cause mortality (Wilmot et al 2012, Biswas et al 2015). Anthropometric measurements such as body mass index (BMI) and waist circumference (WC) have shown conflicting associations with ST (5-7). For example, Van Dyck and colleagues reported that objectively measured ST was not related to BMI after controlling for MVPA in a sample of adults from 12 countries (Dyck et al 2015) while data from the Whitehall II study in the UK revealed no association between self-reported context specific sitting and BMI (Pulsford et al 2013). However, data from NHANES in the US showed that self-reported sitting time was associated with BMI and WC and more so in those who reported no weekly MVPA (Staiano et al 2014). Prospectively, MVPA and ST have been shown to have independent effects on anthropometric and bioimpedance based indices in adults at risk of diabetes (Golubic et al 2014). These discrepancies may be due to errors/limitations in the anthropometric measurements used and often methods such as dual energy x-ray absorptiometry (DXA) are used as a more detailed assessment of regional and whole body adiposity. Less attention has been given to how ST relates to total and regional adiposity and whether any relationships exists independent of objectively measured MVPA.

Along with traditional cardiometabolic risk factors, obesity status is also a key risk factor for type 2 diabetes development (Carey et al 1997). Although typically obesity is assessed using a single, or combination of, anthropometric measurement these measurements cannot distinguish between fat mass and fat free (lean) mass; a decline in the latter is related to functional impairment and mortality while detailed assessment of fat location has shown that trunk fat (deleterious) and leg fat (beneficial) are associated with cardiometabolic risk factors in different directions (Hu et al 2011), As ST has been related to diabetes risk in adults (Wilmot et al 2012, Biswas et al 2015), we aimed to investigate whether objectively measured ST and MVPA are related to total and regional adiposity measured by DXA in adults at risk of type 2 diabetes.

\section{Methods}

\section{Study sample}

The original sample consisted of a convenience sub-sample of adults recruited from 10 primary care practices in Leicestershire, UK for the study 'Walking Away from Diabetes' (Yates et al 2012). This study has been described elsewhere but in brief it was a randomised 
controlled trial (RCT) offering education plus self-monitoring of physical activity for those at risk of type 2 diabetes (Yates et al 2012). Participants were identified as being at high risk of impaired glucose regulation or type 2 diabetes using a modified version of the automated Leicester Diabetes Risk Score (Gray et al 2010) for the primary care setting. Those at or above the 90th percentile of a weighted risk score (using age, sex, ethnicity, BMI, family history of diabetes and medication status) were invited to participate. Ethics and research governance approvals were granted by the Nottingham Research Ethics Committee and Leicestershire, Northamptonshire and Rutland Comprehensive Local Research Network. Written informed consent was obtained from all eligible participants. All measurements were performed by the same team of trained staff who followed identical standard operating procedures.

\section{Participants}

The original sample was 185 adults. The analytical sample in the present analysis was 163 due to exclusion of participants not providing valid accelerometry data $(n=22)$. There were no statistical $(p>0.05)$ differences in age (61.4 versus 64.7 years; $p<0.094)$, BMI (34.5 versus $\left.31.9 \mathrm{~kg} \mathrm{~m}^{-2} ; p<0.095\right)$, sex or ethnicity between participants not providing valid accelerometer than those in the analytical sample.

\section{Total and regional adiposity}

Each subject underwent a DXA scan on a Lunar Prodigy (GE Corporation, USA), software enCORE 2010. DXA utilises a technology by which the attenuation of radiation at 2 energies is used to determine 2 components of the attenuating tissue, either bone and soft tissue or lean soft tissue and fat. DXA has been validated as a measure of body fat in obese and normal weight individuals (Paradisi et al 1999, Bertin et al 2000) and as such is considered to be one of the gold standard measurements for determining body composition. In addition, regional analysis of DXA scans limited to the abdominal area (specifically the region between the top of the first to the bottom of the fourth lumbar vertebrae, L1-L4), has been demonstrated to provide a reasonably valid measure of abdominal adiposity in comparison to CT scans (Svendsen et al 1993). Estimates of total and regional (appendages and trunk) fat mass were obtained, including the abdominal (android) and hip/thigh (gynoid) regions.

\section{Physical activity and sedentary behaviour}

Each participant wore an Actigraph GT3X (Actigraph LLC, Pensacola, Fl, USA) for 7 $\mathrm{d}$ full days during waking hours on the right midaxillary line on an elasticated waistband. Published cut-points (Freedson et al 1998) were applied to the vertical axis activity count data to categorise $15 \mathrm{~s}$ epochs into time spent in MVPA ( $\geqslant 488$ counts $/ 15 \mathrm{~s}$ epoch) and ST $(<25$ counts $/ 15 \mathrm{~s}$ epoch). Breaks in sedentary time (hereafter called 'breaks' (quantified as number of day per day) were also calculated as an interruption in ST that was $\geqslant 25$ counts $/ 15 \mathrm{~s}$ epoch. Accelerometer non-wear time was defined as a minimum of $60 \mathrm{~min}$ of continuous zero counts. Participants with $\geqslant 4$ valid days (a valid day was defined as $\geqslant 600$ min of wear time) were included in the present analysis. All accelerometer-derived variables were computed by summing the values over all valid days and calculating the mean value per valid day. KineSoft version 3.3.76 data analysis tool (Kinesoft, New Brunswick, Canada; available from www. kinesoft.org) was used to process the accelerometer data. 


\section{Covariates}

Age, ethnicity and smoking status were obtained from an interviewer (trained healthcare professional) administered questionnaire.

\section{Statistical analysis}

Multiple regression analysis was used to investigate associations between the accelerometer variables ( min $^{-1}$ of MVPA, and ST and number of breaks $\mathrm{d}^{-1}$ ) with total and regional adiposity variables. Analyses were completed without and with adjustment for known and potential confounders. Model 1 included accelerometer wear time as the only covariate. Model 2 also included age, sex, group (intervention/control), ethnicity, current smoking status, total lean mass, and ST (for MVPA analysis) or MVPA (for both the ST and the breaks analyses). Sex-byvariable (not significant; $p>0.01$ ) and age-by-variable interactions were tested and only the age-by-variable interactions were significant and were retained in the analysis. As the sample was not large enough to stratify by both age and sex groups we decided to stratify by age group only (as often physical activity and sedentary guidelines will be age group and not sex specific). Significance was set at $p<0.05$ (two-tailed) for main effects and $p<0.008$ for interactions. All analyses were completed using SAS v9.4 (SAS Institute Inc., Cary, NC, USA).

\section{Results}

Characteristics of the sample are presented in table 1. This sample being an at risk group was evidenced by $27 \%$ having impaired glucose regulation (having one or both of impaired fasting glucose and impaired glucose tolerance using an oral glucose tolerance test at clinic visit), 36\% having a self-reported family history of diabetes, the sample as a whole spending an average of over $10 \mathrm{~h}$ sedentary per day and $44 \%$ of the sample meeting the recommended $30 \mathrm{~min}^{-1}$ of MVPA.

Table 2 presents the unadjusted and adjusted models for the relationships between ST, breaks and MVPA with the range of adiposity variables. Although ST was positively and significantly associated with total $(b=46.4 ; p=0.002)$, android $(b=5.1 ; p=0.003)$, gynoid $(b=6.4$; $p=0.007), \operatorname{trunk}(b=25.9 ; p=0.005), \operatorname{arm}(b=5.5 ; p=0.002)$ and leg $(b=14.7 ; p=0.005)$ fat mass in the minimally adjusted model, these relationships disappeared after adjustment for MVPA and other key covariates $(p>0.05)$. MVPA was negatively and significantly associated with all variables with total $(b=-617 ; p=0.002)$, android $(b=-70 ; p=0.015)$, gynoid $(b=-85 ; p<0.001)$, trunk $(b=-41 ; p=0.004)$, arm $(b=-56 ; p=0.007)$ and leg $(b=-156 ; p=0.004)$ after adjusting for known and potential confounders, including ST. Except for leg fat mass $(b=94.5 ; p<0.001)$, sedentary breaks were not significantly related to the other adiposity before or after adjustment. However, when breaks were included instead of MVPA as a covariate in the ST analysis, ST still remained significant (results not shown).

The fully adjusted MVPA models for total, android, gynoid and trunk fat showed a significant age by-MVPA interaction $(p<0.008)$ indicating that the relationships between MVPA and these adiposity variables are different at different ages. Table 3 shows the unadjusted and adjusted associations between the adiposity variables and MVPA stratified by age grouping. Similar to the results in table 2, the unadjusted models were significant for all variables for both age groups. However, when ST and other covariates were added to the model the results seen in table 2 were only mirrored for those in the under 65 group. For those in the over 65 group the models did not remain significant when ST and other covariates were added. Mean MVPA was not significantly different $(p=0.33)$ for the younger and older groups was 40.3 (SD 26.6) $\mathrm{min}^{-1}$ and 33.0 (SD 25.4) $\mathrm{min}^{-1}$, respectively. 
Table 1. Participant characteristics of 163 UK adults.

\begin{tabular}{|c|c|}
\hline Males (\%) & $101(62 \%)$ \\
\hline Non-white ethnicity (\%) & $147(10 \%)$ \\
\hline Age (years) & $64.7(7.6)$ \\
\hline BMI $\left(\mathrm{kg} \mathrm{m}^{-2}\right)$ & $31.9(5.9)$ \\
\hline Smokers $(\%)$ & $12(7 \%)$ \\
\hline Family history of diabetes (\%) & $53(36 \%)$ \\
\hline Accelerometer wear time $\left(\operatorname{min~}^{-1}\right)$ & $846(77)$ \\
\hline $\operatorname{MVPA}\left(\operatorname{min~}^{-1}\right)$ & $36(26)$ \\
\hline Sedentary time $\left(\min \mathrm{d}^{-1}\right)$ & $606(81)$ \\
\hline Breaks in sedentary time (breaks $\mathrm{d}^{-1}$ ) & $268(57)$ \\
\hline Total body fat mass $(\mathrm{g})$ & $34846(12110)$ \\
\hline Android fat mass (g) & 3585 (1412) \\
\hline Gynoid fat mass (g) & $4993(1901)$ \\
\hline Trunk fat mass $(\mathrm{g})$ & $20300(7457)$ \\
\hline Arm fat mass $(\mathrm{g})$ & 3615 (1403) \\
\hline Leg fat mass $(\mathrm{g})$ & $9907(4182)$ \\
\hline
\end{tabular}

Note: Continuous variables are presented as means (standard deviation) and ranges and categorical variables presented as number (\% of the total sample). BMI-body mass index; MVPAmoderate to vigorous physical activity.

\section{Discussion}

In this sample of UK adults at risk of type 2 diabetes, ST was not associated to DXA assessed adiposity independent of MVPA and other key covariates. In younger adults (those under 65 years old) at risk of type 2 diabetes, MVPA was negatively related to adiposity independent of ST and other key covariates. However, in adults over 65 year of age the 'good' relationship between MVPA and adiposity was attenuated once ST was taken into account.

The deleterious cardiometabolic effects of ST have been given much attention recently with a new emphasis on the use of a whole spectrum approach to activity related research (Bouchard et al 2015, Lynch and Owen 2015). The impact of ST on adiposity is less clear and is burdened by the variety of methods to assess ST (including the historical use of its proxy TV viewing) and how adiposity is estimated. As mentioned above, anthropometric measurements have showed differing associations with ST even in large international samples (Thorp et al 2009, Pulsford et al 2013, Staiano et al 2014, Dyck et al 2015). Prospective cohort studies have found that higher time spent watching $\mathrm{TV}$ at baseline was associated with elevated risk of obesity (defined using BMI) and type 2 diabetes (Hu et al 2003). Similar to previous analysis of older community dwelling adults (Foong et al 2014) herein we have shown that ST is not related to measures of adiposity using DXA once MVPA and other key covariates, including lean body mass, are taken into account. Therefore, this study suggests that breaks in ST rather than total ST per se may be an important factor in the regulation of body weight. A more robust analysis requires assessment of depot-specific adiposity stores using the gold standard of MRI or CT. Our group has previous shown that ST is indeed related to the specific depots of fat in the heart, liver and visceral region but not with MRI assessed subcutaneous and whole body fat after adjustment for MVPA and glycemia in adults at high risk of type 2 diabetes (Henson et al 2014). Similarly, sitting time has also been associated with CT assessed pericardial fat in older adults (Larsen et al 2014). This would suggest that ST has a different relationship with these ectopic stores.

The significant relationship between MVPA and adiposity is unsurprising as MVPA is recommended for weight control. Work on older adults related MVPA to DXA variables 


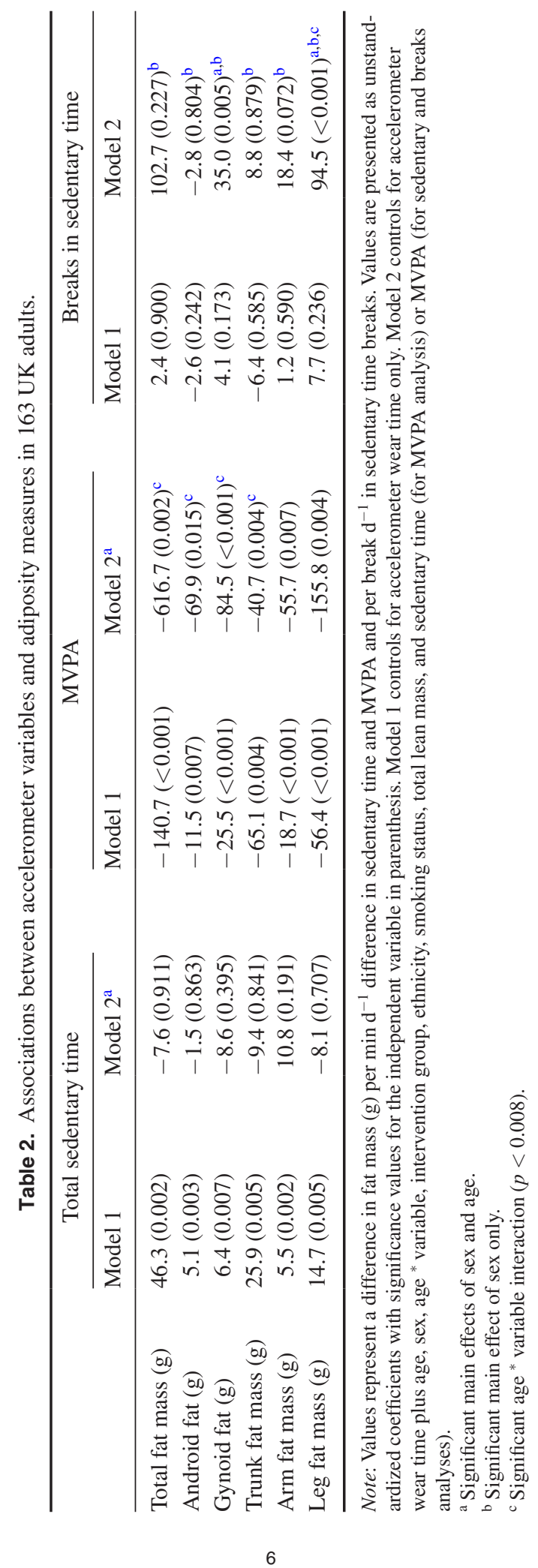


Table 3. Associations between MVPA ( $\operatorname{min~}^{-1}$ ) and adiposity measures in 163 UK adults.

\begin{tabular}{|c|c|c|c|c|}
\hline & \multicolumn{2}{|c|}{$<65$ years old $(n=63)$} & \multicolumn{2}{|c|}{$\geqslant 65$ years old $(n=100)$} \\
\hline & Model 1 & Model 2 & Model 1 & Model 2 \\
\hline $\begin{array}{l}\text { Total fat } \\
\text { mass }(\mathrm{g})\end{array}$ & $-173.4(<0.001)$ & $-215.5(<0.001)$ & $-153.2(<0.001)$ & $-32.3(0.373)$ \\
\hline $\begin{array}{l}\text { Android } \\
\text { fat (g) }\end{array}$ & $-16.6(0.032)$ & $-22.0(0.005)$ & $-11.7(0.007)$ & $-3.8(0.452)$ \\
\hline $\begin{array}{l}\text { Gynoid } \\
\text { fat }(\mathrm{g})\end{array}$ & $-27.8(0.006)$ & $-31.9(<0.001)$ & $-29.2(<0.001)$ & $-7.6(0.182)$ \\
\hline $\begin{array}{l}\text { Trunk fat } \\
\text { mass }(g)\end{array}$ & $-91.6(0.031)$ & $-130.7(0.003)$ & $-66.2(0.002)$ & $-15.8(0.514)$ \\
\hline
\end{tabular}

Note: Values represent a difference in fat mass (grams) per min $\mathrm{d}^{-1}$ change in each independent variable and are presented as unstandardised coefficients with significance values for the independent variable in parenthesis. Model 1 controls for accelerometer wear time only. Model 2 controls for accelerometer wear time plus age, sex, group (intervention/control), ethnicity, current smoking, total lean mass, and sedentary time.

${ }^{a}$ Significant main effects of sex in all models.

(Foong et al 2014) and previous analyses found that MVPA was related to BMI even after ST adjustment (Henson et al 2013, Dyck et al 2015). We have extended these findings using an at risk population recruited from primary care and using an objective assessment of MVPA and a gold standard assessment of adiposity. Breaking up ST has been postulated as a way to negate the negative cardiometabolic effects of sitting too much. This is backed by some epidemiological and experimental evidence (Healy et al 2008, Dunstan et al 2012, Henson et al 2015) although a recent large analysis of older adults with and without diabetes no or weak relationships with cardiometabolic outcomes (van der Berg et al 2016). From a weight control standpoint, epidemiological evidence would suggest that breaks in sitting could be beneficial to the WC of adults who may be at risk of chronic disease (Healy et al 2008) and for body fat levels of healthy older adults (Chastin et al 2012). Similar to the study by Chastain et al (2012) we used the more detailed assessment of adiposity using DXA however their sedentary breaks (called fragmentation of sedentary hours) was assessed by ActivPAL which may be a more valid assessment of breaks than applying cut-points to Actigraph acceleration data (Lyden et al 2012).

Epidemiological evidence from adults over 65 years old in NHANES showed that the deleterious relationship between ST and both BMI and WC existed even in those older adults who were sufficiently active (Gennuso et al 2013). In our sample as a whole (mean age 65 years) we could not corroborate these previous findings. However, the interaction effect of age suggests that the adiposity-related benefits of MVPA in older adults may be reduced due to their engagement in ST. Our finding of an age effect was not seen by Cooper $e t$ al in adults aged 40-69 years who were newly diagnosed with type 2 diabetes (Cooper et al 2014) but was by Foong et al in older adults (mean age 66 years) (Foong et al 2014). Sitting and physical activity have distinct associations with regional fat deposition in older (mean age 65 years) adults (Larsen et al 2014). Bann et al found that relationship between BMI (and not fat mass) and ST in an older sample (mean age $\sim 79$ years old) was as expected (higher ST related to higher BMI) but did not investigate whether this was independent of MVPA due to the low levels of MVPA in their sample (Bann et al 2015). Although there was a $\sim \mathrm{min}^{-1}$ difference in MVPA between the younger and older group herein, the over 65 group still accumulated $33 \mathrm{~min}^{-1}$ of MVPA which is over the recommended level. 
The strengths of this analysis are the objective assessment of physical activity and sedentary behaviour using accelerometry. Gold standard measure of adiposity, high risk population, scans performed by the same individuals according to standardised procedures. Also, this population are ones that have been encouraged to reduce the time they spend sitting in an effort to reduce diabetes risk (National Institute for Health and Care Excellence 2012). The limitations of this analysis are its cross-sectional nature, meaning we cannot infer causality; reverse causality remains a possibility as the relationship between sedentary time and adiposity may be bidirectional, and the relatively small sample size which precluded us from stratifying the analysis by both age and sex may also restrict the external validity of the findings. Although, unlike other studies, we have an objective assessment of ST our device of choice estimates ST based on a lack of movement rather that a direct measure of body posture and does not give a clear distinction between time spent sitting and standing. Accelerometers rely on categorising movement (acceleration), as opposed to distinguishing between specific postures (sitting, lying and standing behaviours), which may lead to an under-estimation of the true association between sedentary time and markers of adiposity. Unmeasured lifestyle measures (particularly snacking) may have also confounded observed relationships.

\section{Conclusion}

Moderate-to-vigorous intensity physical activity is emphasised for weight control and overall good health, particularly in those younger than 65 years at risk of type 2 diabetes. In this sample of adults at risk of type 2 diabetes, MVPA, and not sedentary time, was associated with total and regional adiposity after adjustment for each other. Breaks in ST were not related to adiposity save for leg fat. However, older adults at risk of type 2 diabetes may gain weight related benefits from reducing ST as well as increasing their daily MVPA. Similar to the suggestions by researchers working with people with established type 2 diabetes (Cooper et al 2014, van der Berg et al 2016) and with older adults (Gennuso et al 2013) we recommend that those at risk of type 2 diabetes, particularly older adults, should be supported in becoming and staying physically active at all intensities i.e. increase MVPA and reduce time spent sedentary.

\section{Acknowledgments}

The authors acknowledge support from the National Institute for Health Research Collaboration for Leadership in Applied Health Research and Care-East Midlands (NIHR CLAHRCEM), the Leicester Clinical Trials Unit and the NIHR Leicester-Loughborough Diet, Lifestyle and Physical Activity Biomedical Research Unit, which is a partnership between University Hospitals of Leicester NHS Trust, Loughborough University and the University of Leicester. The views expressed are those of the authors and not necessarily those of the NHS, the NIHR or the Department of Health.

\section{References}

Bann D et al 2015 Light intensity physical activity and sedentary behavior in relation to body mass index and grip strength in older adults: cross-sectional findings from the lifestyle interventions and independence for elders (LIFE) study PloS One 10 e0126063

Bertin E, Marcus C, Ruiz J, Eschard J and Leutenegger M 2000 Measurement of visceral adipose tissue by DXA combined with anthropometry in obese humans Int. J. Obes. $24263-70$ 
Biswas A, Oh P I, Faulkner G E, Bajaj R R, Silver M A, Mitchell M S and Alter D A 2015 Sedentary time and its association with risk for disease incidence, mortality, and hospitalization in adults: a systematic review and meta-analysis Ann. Intern. Med. 162 123-32

Bouchard C, Blair S N and Katzmarzyk P T 2015 Less sitting, more physical activity, or higher fitness? Mayo Clin. Proc. 90 1533-40

Carey V J, Walters E E, Colditz G A, Solomon C G, Willet W C, Rosner B A, Speizer F E and Manson J E 1997 Body fat distribution and risk of non-insulin-dependent diabetes mellitus in women the nurses' health study Am. J. Epidemiol. 145 614-9

Chastin S F M, Ferriolli E, Stephens N A, Fearon K C and Greig C 2012 Relationship between sedentary behaviour, physical activity, muscle quality and body composition in healthy older adults Age Ageing 41 111-4

Cooper A J, Brage S, Ekelund U, Wareham N J, Griffin S J and Simmons R K 2014 Association between objectively assessed sedentary time and physical activity with metabolic risk factors among people with recently diagnosed type 2 diabetes Diabetologia 57 73-82

Dunstan D W et al 2012 Breaking up prolonged sitting reduces postprandial glucose and insulin responses Diabetes Care 35 976-83

Dyck D V et al 2015 International study of objectively measured physical activity and sedentary time with body mass index and obesity: IPEN adult study Int. J. Obes. 39 199-207

Foong Y C, Aitken D, Winzenberg T, Otahal P, Scott D and Jones G 2014 The association between physical activity and reduced body fat lessens with age-results from a cross-sectional study in community dwelling older adults Exp. Gerontol. 55 107-12

Freedson P S, Melanson E and Sirard J 1998 Calibration of the computer science and applications, Inc. accelerometer Med. Sci. Sports Exerc. 30 777-81

Gennuso K P, Gangnon R E, Matthews C E, Thraen-Borowski K M and Colbert L H 2013 Sedentary behavior, physical activity, and markers of health in older adults Med. Sci. Sports Exerc. 45 1493-500

Golubic R, Wijndaele K, Sharp S, Simmons R, Griffin S, Wareham N, Ekelund U and Brage S 2014 Physical activity, sedentary time and gain in overall and central body fat: 7 year follow-up of the ProActive trial cohort Int. J. Obes. $39142-8$

Gray L, Taub N, Khunti K, Gardiner E, Hiles S, Webb D, Srinivasan B and Davies M 2010 The leicester risk assessment score for detecting undiagnosed type 2 diabetes and impaired glucose regulation for use in a multiethnic UK setting Diabet. Med. 27 887-95

Healy G N, Dunstan D W, Salmon J, Cerin E, Shaw J E, Zimmet P Z and Owen N 2008 Breaks in sedentary time beneficial associations with metabolic risk Diabetes Care 31 661-6

Henson J et al 2013 Associations of objectively measured sedentary behaviour and physical activity with markers of cardiometabolic health Diabetologia 1-9

Henson J et al 2014 Associations of sedentary time with fat distribution in a high-risk population Med. Sci. Sports Exerc. 47 1727-34

Henson J et al 2015 Breaking up prolonged sitting with standing or walking attenuates the postprandial metabolic response in postmenopausal women: a randomized acute study Diabetes Care 39 130-8

Hu F B, Li T Y, Colditz G A, Willett W C and Manson J E 2003 Television watching and other sedentary behaviors in relation to risk of obesity and type 2 diabetes mellitus in women J. Am. Med. Assoc. 289 1785-91

Hu G, Bouchard C, Bray G A, Greenway F L, Johnson W D, Newton R L, Ravussin E, Ryan D H and Katzmarzyk P T 2011 Trunk versus extremity adiposity and cardiometabolic risk factors in white and African American adults Diabetes Care 34 1415-8

Larsen B A, Allison M A, Kang E, Saad S, Laughlin G A, Araneta M, Barrett-Connor E and Wassel C L 2014 Associations of physical activity and sedentary behavior with regional fat deposition Med. Sci. Sports Exerc. 46 520-8

Lyden K, Kozey-Keadle S L, Staudenmayer J W and Freedson P S 2012 Validity of two wearable monitors to estimate breaks from sedentary time Med. Sci. Sports Exerc. $442243-52$

Lynch B M and Owen N 2015 Too much sitting and chronic disease risk: steps to move the science forward Ann. Intern. Med. 162 146-7

National Institute for Health and Care Excellence 2012 Type 2 Diabetes: Prevention in People at High Risk vol PH38 (Manchester: National Institute for Health and Care Excellence)

Paradisi G et al 1999 Dual energy x-ray absorptiometry assessment of fat mass distribution and its association with the insulin resistance syndrome Diabetes Care 22 1310-7

Pulsford R M, Stamatakis E, Britton A R, Brunner E J and Hillsdon M M 2013 Sitting behavior and obesity: evidence from the Whitehall II study Am. J. Prev. Med. 44 132-8 
Staiano A, Harrington D, Barreira T and Katzmarzyk P 2014 Sitting time and cardiometabolic risk in US adults: associations by sex, race, socioeconomic status and activity level Br. J. Sports Med. 48 213-9

Svendsen O, Hassager C, Bergmann I and Christiansen C 1993 Measurement of abdominal and intraabdominal fat in postmenopausal women by dual energy $\mathrm{x}$-ray absorptiometry and anthropometry: comparison with computerized tomography Int. J. Obes. 17 45-51

Thorp A A, Healy G N, Owen N, Salmon J, Ball K, Shaw J E, Zimmet P Z and Dunstan D W 2009 Deleterious associations of sitting time and television viewing time with cardio-metabolic risk biomarkers: AusDiab 2004-2005 Diabetes Care

van der Berg J D et al 2016 Associations of total amount and patterns of sedentary behaviour with type 2 diabetes and the metabolic syndrome: the maastricht study Diabetologia 59 709-18

Wilmot E G, Edwardson C L, Achana F A, Davies M J, Gorely T, Gray L J, Khunti K, Yates T and Biddle S J 2012 Sedentary time in adults and the association with diabetes, cardiovascular disease and death: systematic review and meta-analysis Diabetologia 55 2895-905

Yates T, Davies M J, Henson J, Troughton J, Edwardson C, Gray L J and Khunti K 2012 Walking away from type 2 diabetes: trial protocol of a cluster randomised controlled trial evaluating a structured education programme in those at high risk of developing type 2 diabetes BMC Family Pract. 1346 


\section{QUERIES}

\section{Page 2}

AQ1

Please be aware that the colour figures in this article will only appear in colour in the online version. If you require colour in the printed journal and have not previously arranged it, please contact the Production Editor now.

Page 7

AQ2

Please provide the significance of [a] in table [3].

Page 8

AQ3

Please check the details for any journal references that do not have a link as they may contain some incorrect information.

Page 9

AQ4

Please provide the volume for Henson et al (2013).

Page 10

AQ5

Please update the volume and page range/article number in Thorp et al (2009). 